\title{
Optical position meters analyzed in the non-inertial reference frames
}

\author{
Sergey P. Tarabrin and Alexander A. Seleznyov \\ Faculty of Physics, Moscow State University, Moscow, 119992, Russit*
}

(Dated: October 30, 2018)

\begin{abstract}
In the framework of General Relativity we develop a method for analysis of the operation of the optical position meters in their photodetectors proper reference frames. These frames are noninertial in general due to the action of external fluctuative forces on meters test masses, including detectors. For comparison we also perform the calculations in the laboratory (globally inertial) reference frame and demonstrate that for certain optical schemes laboratory-based analysis results in unmeasurable quantities, in contrast to the detector-based analysis. We also calculate the response of the simplest optical meters to weak plane gravitational waves and fluctuative motions of their test masses. It is demonstrated that for the round-trip meter analysis in both the transversetraceless (TT) and local Lorentz (LL) gauges produces equal results, while for the forward-trip meter corresponding results differ in accordance with different physical assumptions (e.g. procedure of clocks synchronization) implicitly underlying the construction of the TT and LL gauges.

PACS numbers: 04.30.Nk, 04.80.Nn, 07.60.Ly, 95.55.Ym
\end{abstract}

\section{INTRODUCTION}

Optical position meters can be thought of as constituent parts of laser interferometers such as Michelson or Mach-Zehnder. For instance, long-baseline Michelson interferometer is the composition of two round-trip position meters: two optical waves, emerging from and returning to approximately the same spacial point, inside interferometer arms carry the information about displacements of the end-mirrors. Upon their arrival to photodetector the latter measures the relative phase of two waves proportional to the relative displacement of the end-mirrors.

Currently long-baseline optical interferometers, such as LIGO [1], are the world most sensitive instruments for measuring the relative displacements between the test masses, which might be produced by the gravitational waves (GWs) coming to the Earth from astrophysical sources. Traditionally in literature interaction of the gravitational waves with laser interferometers is considered in the framework of the so-called transverse-traceless (TT) gauge [2, 3, 4]. Though the TT-based analysis of the GW detectors is usually exceptionally simple, it is sometimes hard to be justified physically since the major requirement of the TT gauge is the ideal inertiality of the test masses. In other words, in order to validate the TT-based analysis one must require that the test masses strictly follow the geodesics of the GW space-time, i.e. no non-gravitational forces are present. However, at least in the Earth-bound experimental installations test masses undergo various non-geodesic motions due to noises in the environment (such as seismic noise), in the test masses themselves (thermal noise), due to measurement devices (back-action noise) and others.

In order to deal correctly with external forces when

*Electronic address: tarabrin@phys.msu.ru analyzing the operation of an interferometer (or optical position meter) one should perform the calculations in the proper reference frame of its detector since it is the device that produces an experimentally observable quantity. Since detector is subjected to the action of fluctuative forces in general, its proper reference frame is non-inertial. This is particularly important for the certain class of the GW detectors called displacement-noisefree interferometers, which are mostly of Mach-Zehnder [5, 6, 7, 8] or Fabry-Perot [9] type, where non-geodesic motion of detector(s) may significantly affect the operation of an interferometer limiting its sensitivity to GWs. The main goal of this paper is to develop the method of solving certain electrodynamical problems in such noninertial reference frames.

\section{SPACE-TIME IN THE VICINITY OF AN ACCELERATED OBSERVER}

\section{A. Metric tensor}

We start from the introduction of space-time metric in the vicinity of an accelerated observer. First, consider the laboratory (globally inertial) frame with Minkowski metric $d s^{2}=\eta_{\alpha \beta} d x^{\alpha} d x^{\beta}$, where $\eta_{\alpha \beta}=\operatorname{diag}(-1,1,1,1)$. Greek indices run over $0,1,2,3$.

It is known that the non-relativistic $\left(v^{2} / c^{2} \ll 1\right)$ coordinate transformation 10

$$
x=x^{\prime}+\frac{a_{x}\left(t^{\prime}\right) t^{\prime 2}}{2}, \quad t=t^{\prime}\left[1+\frac{a_{x}\left(t^{\prime}\right) x^{\prime}}{c^{2}}\right] .
$$

brings us from the laboratory frame to the proper reference frame of the observer (reference body for definiteness) moving with acceleration $a_{x}\left(t^{\prime}\right)$ along the $x$-axis. Throughout the paper prime denotes the physical quantity evaluated in the observer's proper reference frame. According to the transformation law, metric in the vicinity of an accelerated observer, accurate to linear order of 
$x^{\prime}$, takes the form:

$$
\begin{aligned}
d s^{2} & =g_{\alpha \beta}\left(x^{\prime \mu}\right) d x^{\prime \alpha} d x^{\prime \beta} \\
& =-\left(c d t^{\prime}\right)^{2}\left[1+\frac{2}{c^{2}} a_{x}\left(t^{\prime}\right) x^{\prime}\right]+d x^{\prime 2}+d y^{\prime 2}+d z^{\prime 2}
\end{aligned}
$$

We assume that the acceleration $a_{x}\left(t^{\prime}\right)$ is so small that for all $x^{\prime}$ and $t^{\prime}$ condition $\left|2 a_{x}\left(t^{\prime}\right) x^{\prime} / c^{2}\right| \ll 1$ is fulfilled. In particular, for the ground-based interferometers one can estimate $a_{x} \lesssim \Omega_{\max }^{2} \xi$ with $\Omega_{\max } \sim 10^{3} \mathrm{~Hz}$ (the upper boundary of the operating frequency band), $\xi \sim 10^{-19} \mathrm{~m}$ (typical values of the fluctuative displacements), $x \sim 10^{3} \mathrm{~m}$ (typical interferometer arm length), so that $\left|2 a_{x} x^{\prime} / c^{2}\right| \sim 10^{-24}$. Therefore, we will use the methods of linearized theory in full similarity with the theory of linear gravitational waves; we will keep only the 0 th and the 1st order in $a_{x}\left(t^{\prime}\right)$ terms further.

Metric tensor corresponding to interval (2) and the determinant of its matrix are:

$$
\begin{gathered}
g_{\alpha \beta}=\left(\begin{array}{cccc}
-1-2 a_{x}\left(t^{\prime}\right) x^{\prime} / c^{2} & 0 & 0 & 0 \\
0 & 1 & 0 & 0 \\
0 & 0 & 1 & 0 \\
0 & 0 & 0 & 1
\end{array}\right), \\
g=\operatorname{det}\left(g_{\alpha \beta}\right)=-1-\frac{2}{c^{2}} a_{x}\left(t^{\prime}\right) x^{\prime} .
\end{gathered}
$$

\section{B. Test masses equation of motion}

Since we consider the motion of the test masses along the $x^{\prime}$-axis, the only Christoffel symbols needed are $\Gamma_{\nu \lambda}^{\mu}$ with $\mu=1: \Gamma_{00}^{1}=a_{x}\left(t^{\prime}\right) / c^{2}, \Gamma_{01}^{1}=0$. Geodesic equation for the $x$-axis reads:

$$
\frac{d^{2} x^{\prime 1}}{d s^{2}}+\Gamma_{00}^{1}\left(\frac{d x^{\prime 0}}{d s}\right)^{2}+2 \Gamma_{01}^{1} \frac{d x^{\prime 0}}{d s} \frac{d x^{\prime 1}}{d s}+\Gamma_{11}^{1}\left(\frac{d x^{\prime 1}}{d s}\right)^{2}=0 .
$$

In the non-relativistic approximation $d s \approx c d t^{\prime}$ and $\left(d x^{1} / d s\right)^{2} \approx\left(v^{\prime} / c\right)^{2} \ll 1$, therefore we obtain the following equation of motion (for strict derivation see Ref. [11]):

$$
\frac{d^{2} x^{\prime}}{d t^{\prime 2}}=-a_{x}\left(t^{\prime}\right)
$$

which coincides exactly with the Newtonian law of motion in the non-inertial frame. If the test mass $m$ is also subjected to some external force $F_{x}\left(t^{\prime}\right)$ as seen from the laboratory frame, then the latter should be added to the right side of the equation:

$$
\frac{d^{2} x^{\prime}}{d t^{\prime 2}}=-a_{x}\left(t^{\prime}\right)+\frac{F_{x}\left(t^{\prime}\right)}{m} .
$$

Thus, in the absence of the observer's acceleration (when $x=x^{\prime}$ and $t=t^{\prime}$ ) we obtain the Newtonian motion law of the test mass in the laboratory frame: $d^{2} x / d t^{2}=$ $F_{x}(t) / m$.
For simplicity we assume that the test mass and the observer stay in rest, separated by a distance $x_{0}^{\prime}=x_{0}=$ const, with respect to the laboratory frame in the absence of all forces in order not to consider the effects of uniform motion.

Below we will consider the problems where test masses undergo tiny fluctuative displacements under the influence of external forces, i.e. $F_{x} / m$ is of the same order of smallness as $a_{x}$. Thus, Eq. (44) allows significant simplification: according to the transformation law (1) coordinate time in the observer's frame $t^{\prime}$ and the one in the laboratory frame $t$ differ in the amount proportional to $a_{x} x^{\prime} / c^{2}$ which is the quantity of the 1 st order of smallness. Therefore, up to the 1st order $a_{x}\left(t^{\prime}\right)=a_{x}(t)$ and $F_{x}\left(t^{\prime}\right)=F_{x}(t)$. Under the listed assumptions Eq. (4) can be integrated in the following form:

$$
x^{\prime}(t)=x_{0}+\int_{-\infty}^{t} d t_{1} \int_{-\infty}^{t_{1}} d t_{2}\left[-a_{x}\left(t_{2}\right)+\frac{F_{x}\left(t_{2}\right)}{m}\right] .
$$

It will be convenient to separate the 0th order and the 1st order summands: $x^{\prime}(t)=x_{0}+\delta x^{\prime}(t),\left|\delta x^{\prime}\right| \ll\left|x_{0}\right|$,

$$
\delta x^{\prime}(t)=\xi(t)-\xi_{\mathrm{ref}}(t),
$$

where $\xi(t)$ is the result of double integration of $F_{x}(t) / m$ and $\xi_{\text {ref }}(t)$ is the result of double integration of $a_{x}(t)$. The physical meaning of these quantities is clear: $\xi$ and $\xi_{\text {ref }}$ are the displacements of the test mass and the reference mass correspondingly with respect to the laboratory frame, while $\delta x^{\prime}$ is the displacement of the test mass with respect to the reference mass (i.e. with respect to the proper reference frame of the reference mass).

\section{ELECTROMAGNETIC WAVE IN THE SPACE-TIME OF AN ACCELERATED OBSERVER}

In the interferometric experiments an observer studies the motion of the test masses by sending and receiving the reflected light waves. According to the equivalence principle an accelerated frame (of the observer) is equivalent to some gravitational field which is known to impose the distributed redshift on electromagnetic waves. Thus it is necessary to calculate the propagation of electromagnetic waves in the space-time of an accelerated observer in order to obtain a complete description of an interferometer.

\section{A. Wave equation}

We will derive the wave equation from the second pair of Maxwell's equations without the sources:

$$
\frac{1}{\sqrt{-g}} \frac{\partial}{\partial x^{\prime \beta}}\left(\sqrt{-g} F^{\alpha \beta}\right)=0 .
$$


Here $F_{\mu \nu}^{\prime}=\partial_{\mu}^{\prime} A_{\nu}^{\prime}-\partial_{\nu}^{\prime} A_{\mu}^{\prime}$ and $A^{\prime \mu}=\left(A^{\prime 0}, A^{\prime 1}, A^{\prime 2}, A^{\prime 3}\right)$ is the 4-potential of electromagnetic field. Substituting the definition of $F_{\mu \nu}^{\prime}$ into the field equations we obtain:

$$
\begin{aligned}
\left(\partial_{\beta}^{\prime} \sqrt{-g}\right)\left(\partial^{\prime \alpha} A^{\prime \beta}-\partial^{\prime \beta} A^{\prime \alpha}\right) & \\
& +\sqrt{-g}\left(\partial^{\prime \alpha} \partial_{\beta}^{\prime} A^{\prime \beta}-\partial_{\beta}^{\prime} \partial^{\prime \beta} A^{\prime \alpha}\right)=0 .
\end{aligned}
$$

Let us impose Lorentz or Coulomb gauge (this will influence only the procedure of quantization) so that $\partial_{\beta}^{\prime} A^{\prime \beta}$ vanishes. Remind now that the non-zero components of metric tensor (31) are $g_{00}, g_{11}, g_{22}$ and $g_{33}$ and let us assume that the vector-potential describes the propagation along the $x^{\prime}$-axis of the plane electromagnetic wave polarized along the $z^{\prime}$-axis, i.e. $A^{\prime \alpha}=\left(0,0,0, A^{\prime}\right)$ and $A^{\prime}=A^{\prime}\left(x^{\prime 0}, x^{\prime 1}\right)=A^{\prime}\left(x^{\prime}, t^{\prime}\right)$. Remind also that $g=g\left(x^{\prime}, t^{\prime}\right)$. Therefore, wave equation reduces to:

$$
\begin{aligned}
& \left(\partial_{0}^{\prime} \sqrt{-g}\right)\left(-g^{00} \partial_{0}^{\prime} A^{\prime}\right)+\left(\partial_{1}^{\prime} \sqrt{-g}\right)\left(-g^{11} \partial_{1}^{\prime} A^{\prime}\right) \\
& -\sqrt{-g}\left(g^{00} \partial_{0}^{\prime} \partial_{0}^{\prime} A^{\prime}+g^{11} \partial_{1}^{\prime} \partial_{1}^{\prime} A^{\prime}\right)=0 .
\end{aligned}
$$

Substituting here the components of metric tensor in an explicit form and linearizing the equation with respect to the terms containing $a_{x}\left(t^{\prime}\right)$, we finally obtain the following scalar wave equation:

$$
\begin{aligned}
\frac{1}{c^{2}} \frac{\partial^{2} A^{\prime}}{\partial t^{\prime 2}}-\frac{\partial^{2} A^{\prime}}{\partial x^{\prime 2}}= & \frac{a_{x} x^{\prime}}{c^{2}}\left(\frac{1}{c^{2}} \frac{\partial^{2} A^{\prime}}{\partial t^{\prime 2}}+\frac{\partial^{2} A^{\prime}}{\partial x^{\prime 2}}\right) \\
& -\frac{\dot{a}_{x} x^{\prime}}{c^{3}} \frac{1}{c} \frac{\partial A^{\prime}}{\partial t^{\prime}}+\frac{a_{x}}{c^{2}} \frac{\partial A^{\prime}}{\partial x^{\prime}} .
\end{aligned}
$$

Here $\dot{a}_{x}=d a_{x}\left(t^{\prime}\right) / d t^{\prime}$. The right side of this equation describes the redshift produced by the non-inertiality of the reference frame.

\section{B. Solution of the wave equation}

It is convenient to solve the obtained equation using the method of successive approximations in full similarity with the solution of wave equation in Ref [12]. We shall keep only the 0th and the 1st order in $a_{x}\left(t^{\prime}\right)$ terms: $A^{\prime}\left(x^{\prime}, t^{\prime}\right)=A^{\prime(0)}\left(x^{\prime}, t^{\prime}\right)+A^{\prime(1)}\left(x^{\prime}, t^{\prime}\right), \quad\left|A^{\prime(1)}\right| \sim$ $\left|\left(a_{x} x^{\prime} / c^{2}\right) A^{\prime(0)}\right| \ll\left|A^{\prime(0)}\right|$. The 0th order corresponds to the unaccelerated observer which stays in rest in the laboratory frame. Thus, solution of the 0th order can be represented as a sum of plane monochromatic waves traveling in positive and negative directions of the $x^{\prime}$-axis with amplitudes and frequency measured in the laboratory frame in the state of rest. We denote "positive" wave with index '+' and "negative" wave with index '-':

$$
\begin{aligned}
& A^{\prime(0)}\left(x^{\prime}, t^{\prime}\right)=A_{+}^{\prime(0)}\left(x^{\prime}, t^{\prime}\right)+A_{-}^{\prime(0)}\left(x^{\prime}, t^{\prime}\right), \\
& A_{ \pm}^{\prime(0)}=A_{ \pm 0} e^{-i\left(\omega_{0} t^{\prime} \mp k_{0} x^{\prime}\right)}+\text { c.c. },
\end{aligned}
$$

where $k_{0}=\omega_{0} / c$. Evidently, in the 0th order $t^{\prime}=t$ and $x^{\prime}=x$. Amplitudes and frequency are derived from some initial and boundary problems and we shall keep them undefined until next section. The 1st order equation is:

$$
\begin{aligned}
\frac{1}{c^{2}} \frac{\partial^{2} A_{ \pm}^{\prime(1)}}{\partial t^{\prime 2}}-\frac{\partial^{2} A_{ \pm}^{\prime(1)}}{\partial x^{\prime 2}}= & \frac{a_{x} x^{\prime}}{c^{2}}\left(\frac{1}{c^{2}} \frac{\partial^{2} A_{ \pm}^{\prime(0)}}{\partial t^{\prime 2}}+\frac{\partial^{2} A_{ \pm}^{\prime(0)}}{\partial x^{\prime 2}}\right) \\
& -\frac{\dot{a}_{x} x^{\prime}}{c^{3}} \frac{1}{c} \frac{\partial A_{ \pm}^{\prime(0)}}{\partial t^{\prime}}+\frac{a_{x}}{c^{2}} \frac{\partial A_{ \pm}^{\prime(0)}}{\partial x^{\prime}}
\end{aligned}
$$

The general solution of this equation can be represented as a sum of "positive" and "negative" waves:

$$
A^{\prime(1)}\left(x^{\prime}, t^{\prime}\right)=A_{+}^{\prime(1)}\left(x^{\prime}, t^{\prime}\right)+A_{-}^{\prime(1)}\left(x^{\prime}, t^{\prime}\right) .
$$

Clearly, they can be treated independently. Remind, that $g_{00}(0, t)=-1$ and therefore, we must demand that

$$
A_{+}^{\prime(1)}\left(0, t^{\prime}\right)=A_{-}^{\prime(1)}\left(0, t^{\prime}\right)=0 .
$$

Physically these initial conditions mean that both the light waves $A_{ \pm}^{\prime}\left(x^{\prime}, t^{\prime}\right)$ experience no redshift at $x^{\prime}=0$, i.e. the solution of full Eq. (77) at $x^{\prime}=0$ is $A_{ \pm}^{\prime}\left(0, t^{\prime}\right)=$ $A_{ \pm}^{\prime(0)}\left(0, t^{\prime}\right)=A_{ \pm 0} e^{-i \omega_{0} t}+A_{ \pm 0}^{*} e^{i \omega_{0} t}$ (remind, that $t^{\prime}=t$ at $x^{\prime}=0$ according to Eqs. (10). The solution of Cauchy problem (9-11) is obtained in Appendix A. For slow enough mechanical motions (as compared to the optical frequency) we have:

$A_{ \pm}^{\prime(1)}\left(x^{\prime}, t^{\prime}\right)=A_{ \pm 0} w_{ \pm}^{\prime}\left(x^{\prime}, t^{\prime}\right) e^{-i\left(\omega_{0} t^{\prime} \mp k_{0} x^{\prime}\right)}+$ c.c.,

$w_{ \pm}^{\prime}\left(x^{\prime}, t^{\prime}\right)=-i k_{0} \dot{\xi}_{\text {ref }}\left(t^{\prime}\right) \frac{x^{\prime}}{c} \pm i k_{0}\left[\xi_{\text {ref }}\left(t^{\prime}\right)-\xi_{\text {ref }}\left(t^{\prime} \mp x^{\prime} / c\right)\right]$.

Remind, that $\xi_{\text {ref }}\left(t^{\prime}\right)$ is the result of integration of $a_{x}\left(t^{\prime}\right)$ such that $a_{x}\left(t^{\prime}\right)=\ddot{\xi}_{\text {ref }}\left(t^{\prime}\right)$. Throughout the paper below we will omit the "c.c." notation for briefness.

Similarly to the previous section we may replace $t^{\prime} \rightarrow t$ and $x^{\prime} \rightarrow x$ in $w^{\prime}\left(x^{\prime}, t^{\prime}\right)$ without introducing an error in the 1st order:

$$
w_{ \pm}^{\prime}(x, t)=-i k_{0} \dot{\xi}_{\text {ref }}(t) \frac{x}{c} \pm i k_{0}\left[\xi_{\text {ref }}(t)-\xi_{\text {ref }}(t \mp x / c)\right] .
$$

Whenever it is convenient we will use either $w_{ \pm}^{\prime}\left(x^{\prime}, t^{\prime}\right)$ or $w_{ \pm}^{\prime}(x, t)$ below.

Several features of $w_{ \pm}^{\prime}(x, t)$ are worth noting. First, according to the physical sense $w_{ \pm}(0, t)=w_{ \pm}\left(0, t^{\prime}\right)=0$, i.e. the frequency of electromagnetic wave is not redshifted in the immediate vicinity of an observer. Second, for small enough $x / c$ (in spectral domain this corresponds to $\Omega x / c \ll 1$ limit) $w_{ \pm}^{\prime}(x, t)$ has the $O\left[k_{0}(x / c)^{2} a_{x}(t)\right]$ asymptotic. This agrees with the relativity principle: electromagnetic wave senses only the acceleration of the reference frame, not the displacement $\xi_{\text {ref }}$ or velocity $\dot{\xi}_{\text {ref }}$. Finally, since $\xi_{\text {ref }}(t)$ and $\dot{\xi}_{\text {ref }}(t)$ are the pure real quantities, $w_{ \pm}^{\prime}(x, t)$ are the pure imaginary quantities and therefore describe the influence of the acceleration on the phase (amplitude is affected beginning from the 2nd order):

$$
\begin{aligned}
A_{ \pm}^{\prime}\left(x^{\prime}, t^{\prime}\right) & =A_{ \pm 0}\left[1+w_{ \pm}^{\prime}\left(x^{\prime}, t^{\prime}\right)\right] e^{-i\left(\omega_{0} t^{\prime} \mp k_{0} x^{\prime}\right)} \\
& \approx A_{ \pm 0} e^{-i\left(\omega_{0} t^{\prime} \mp k_{0} x^{\prime}\right)+w_{ \pm}^{\prime}\left(x^{\prime}, t^{\prime}\right)}
\end{aligned}
$$




\section{ROUND-TRIP POSITION METER}

Let us consider now the optical scheme of the roundtrip position meter illustrated in Fig. 1) light wave emitted by the laser mounted on test mass $a$ reaches the absolutely reflective mirror (test mass $b$ ) and is reflected back to the detector mounted on test mass $a$. We are interested in the phase shift acquired by the light wave. Such a position meter might be a constituent part (a single arm) of a Michelson interferometer. In the state of rest the distance between the test masses equals to $L$.

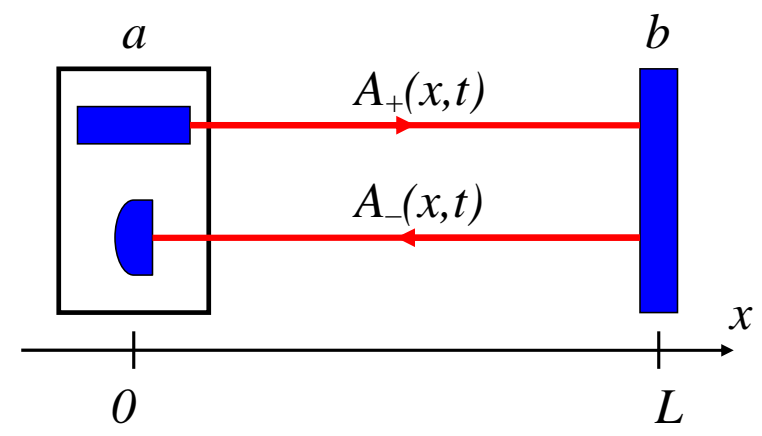

FIG. 1: A round-trip position meter. Laser mounted on test mass $a$ emits the wave which is reflected from the movable mirror $b$. Detector mounted on test mass $a$ detects the reflected wave and measures the acquired phase shift. In the state of rest the distance between the test masses equals to $L$.

\section{A. Analysis in the inertial frame}

First we analyze the situation from the viewpoint of observer in the laboratory (globally inertial) reference frame. Coordinates of the test masses $a$ and $b$ are $x_{a}(t)=0+\xi_{a}(t)$ and $x_{b}(t)=L+\xi_{b}(t)$ correspondingly. Remind that $\left|\xi_{a, b}\right| \ll L$. We also approximate the light wave as noiseless (see Appendix B for generalization). Thus we may write the wave that laser emits in the following form:

$$
A_{+}(x, t)=A_{+0} \exp \left\{-i \omega_{0}\left[t-\frac{x-x_{a}(t-x / c)}{c}\right]\right\},
$$

The wave reflected from the mirror is described by vectorpotential

$$
A_{-}(x, t)=A_{-0} e^{-i\left(\omega_{0} t+k_{0} x\right)}+a_{-}(x, t) e^{-i\left(\omega_{0} t+k_{0} x\right)},
$$

where $a_{-}(x, t)$, which carries the information about the acquired phase shift, can be represented as the Fourier integral:

$$
a_{-}(x, t)=\int_{-\infty}^{+\infty} a_{-}\left(\Omega+\omega_{0}\right) e^{-i \Omega(t+x / c)} \frac{d \Omega}{2 \pi} .
$$

To find the relationship between the incident and the reflected waves we impose the boundary condition which states that the vector potential vanishes on the mirror surface:

$$
A_{+}\left(x_{b}(t), t\right)+A_{-}\left(x_{b}(t), t\right)=0 .
$$

Substituting fields (13) and (14) into this equation and keeping only the 0 th and the 1st order terms we obtain:

$$
\begin{aligned}
& A_{+0} e^{i k_{0} L}\left[1+i k_{0} \xi_{b}(t)-i k_{0} \xi_{a}(t-\tau)\right] \\
& \quad+A_{-0} e^{-i k_{0} L}\left[1-i k_{0} \xi_{b}(t)\right]+a_{-}(L, t) e^{-i k_{0} L}=0 .
\end{aligned}
$$

Here $\tau=L / c$. The 0 th order solution is

$$
A_{-0}=-A_{+0} e^{2 i \omega_{0} \tau}
$$

In the 1st order we obtain:

$$
a_{-}(L, t)=-A_{+0} e^{2 i \omega_{0} \tau} i k_{0}\left[2 \xi_{b}(t)-\xi_{a}(t-\tau)\right],
$$

or

$$
a_{-}(0, t)=-A_{+0} e^{2 i \omega_{0} \tau} i k_{0}\left[2 \xi_{b}(t-\tau)-\xi_{a}(t-2 \tau)\right],
$$

according to the wave-like representation of $a_{-}(x, t)$.

Detection of the reflected wave takes place at point $x=$ $x_{a}(t)=\xi_{a}(t)$. Therefore, total variation of the optical wave $\delta a(t)$ per round trip equals to $a_{-}(0, t)-A_{-0} i k_{0} \xi_{a}(t)$ :

$$
\delta a(t)=-A_{+0} e^{2 i \omega_{0} \tau} i k_{0}\left[2 \xi_{b}(t-\tau)-\xi_{a}(t-2 \tau)-\xi_{a}(t)\right] .
$$

We are interested in the phase shift $\delta \Psi$ described by the term in the square brackets:

$$
\delta \Psi(t)=-k_{0}\left[\xi_{a}(t)-2 \xi_{b}(t-\tau)+\xi_{a}(t-2 \tau)\right] .
$$

Evidently, this phase shifts describes a round trip of light wave with correct time delays. If the entire system moves as a rigid body, i.e. $\xi_{a}(t)=\xi_{b}(t)$, then $\delta \Psi(t) \approx-k_{0} \ddot{\xi}_{a}(t) \tau^{2}$. This result agrees with the relativity principle: no absolute displacement $\xi_{a}$ or velocity $\dot{\xi}_{a}$ can be measured.

\section{B. Analysis in the non-inertial frame}

Now we will consider the same situation in the proper reference frame of the test mass $a$, where detector is mounted, and compare the result with the one of the laboratory-frame analysis.

Since test mass $a$ is the reference body, its equation of motion is $x_{a}^{\prime}(t)=0$. The coordinate of test mass $b$ is $x_{b}^{\prime}(t)=L+\delta x_{b}^{\prime}(t)$ with $\left|\delta x_{b}^{\prime}\right| \ll L$.

In its proper reference frame laser emits the wave described by vector-potential

$$
A_{+}^{\prime}\left(x^{\prime}, t^{\prime}\right)=A_{+0}\left[1+w_{+}^{\prime}\left(x^{\prime}, t^{\prime}\right)\right] e^{-i\left(\omega_{0} t^{\prime}-k_{0} x^{\prime}\right)},
$$


according to the results of Sec. IIIB. Reflected wave in the reference frame of test mass $a$ is described by vectorpotential

$$
\begin{aligned}
A_{-}^{\prime}\left(x^{\prime}, t^{\prime}\right)= & A_{-0}\left[1+w_{-}^{\prime}\left(x^{\prime}, t^{\prime}\right)\right] e^{-i\left(\omega_{0} t^{\prime}+k_{0} x^{\prime}\right)} \\
& +a_{-}^{\prime}\left(x^{\prime}, t^{\prime}\right) e^{-i\left(\omega_{0} t^{\prime}+k_{0} x^{\prime}\right)}
\end{aligned}
$$

Here $a_{-}^{\prime}\left(x^{\prime}, t^{\prime}\right)$ has the same physical meaning as in the previous section and thus has the 1st order of smallness.

Substituting both waves into the boundary condition

$$
A_{+}^{\prime}\left(x_{b}^{\prime}\left(t^{\prime}\right), t^{\prime}\right)+A_{-}\left(x_{b}^{\prime}\left(t^{\prime}\right), t^{\prime}\right)=0 .
$$

we obtain:

$$
\begin{aligned}
& A_{+0} e^{i k_{0} L}\left[1+i k_{0} \delta x_{b}^{\prime}(t)+w_{+}^{\prime}(L, t)\right] \\
& +A_{-0} e^{-i k_{0} L}\left[1-i k_{0} \delta x_{b}^{\prime}(t)+w_{-}^{\prime}(L, t)\right] \\
& +a_{-}^{\prime}(L, t) e^{-i k_{0} L}=0 .
\end{aligned}
$$

The 0th order solution is similar to the previous case: $A_{-0}=-A_{+0} e^{2 i \omega_{0} \tau}$. In the 1st order we obtain:

$$
\begin{aligned}
& a_{-}^{\prime}(0, t)=-A_{+0} e^{2 i \omega_{0} \tau} \\
& \quad \times\left[2 i k_{0} \delta x_{b}^{\prime}(t-\tau)+w_{+}^{\prime}(L, t-\tau)-w_{-}^{\prime}(L, t-\tau)\right] .
\end{aligned}
$$

Since detection of the reflected wave takes place at point $x^{\prime}=x_{a}^{\prime}(t)=0$ in the reference frame we work in, total variation of the optical wave coincides with $a_{-}^{\prime}(0, t)$. Phase shift describing the round trip is:

$$
\begin{aligned}
& i \delta \Psi^{\prime}(t) \\
& =\left[2 i k_{0} \delta x_{b}^{\prime}(t-\tau)+w_{+}^{\prime}(L, t-\tau)-w_{-}^{\prime}(L, t-\tau)\right] \\
& =2 i k_{0} \delta x_{b}^{\prime}(t-\tau)-i k_{0}\left[\xi_{a}(t)-2 \xi_{a}(t-\tau)+\xi_{a}(t-2 \tau)\right] .
\end{aligned}
$$

To analyze its physical meaning we first expand it in the series of $\tau$ keeping all the terms up to $\ddot{\xi}_{a} \tau^{2}$ :

$$
\delta \Psi^{\prime}(t) \approx 2 k_{0} \delta x_{b}^{\prime}(t)-k_{0} \ddot{\xi}_{a}(t) \tau^{2} .
$$

If the entire system moves as a rigid body, i.e. $\delta x_{b}^{\prime}(t)=$ 0 , then $\delta \Psi^{\prime}(t) \approx-k_{0} \ddot{\xi}_{a}(t) \tau^{2}$ in full agreement with the relativity principle.

Let us now substitute solution (6) for $\delta x_{b}^{\prime}$ into the phase shift (15), keeping in mind that $\xi_{\text {ref }}=\xi_{a}$ and $\xi=\xi_{b}$ :

$$
\begin{aligned}
\delta \Psi^{\prime}(t)= & 2 k_{0}\left[\xi_{b}(t-\tau)-\xi_{a}(t-\tau)\right] \\
& -k_{0}\left[\xi_{a}(t)-2 \xi_{a}(t-\tau)+\xi_{a}(t-2 \tau)\right] \\
= & -k_{0}\left[\xi_{a}(t)-2 \xi_{b}(t-\tau)+\xi_{a}(t-2 \tau)\right] .
\end{aligned}
$$

Thus, the obtained phase shift $\delta \Psi^{\prime}(t)$ coincides with the phase shift $\delta \Psi(t)$ in the laboratory frame. In other words, consideration in both the frames results in equal measurable quantities. This coincidence owes to the fact that in our round-trip scheme phases of both the emitted and reflected waves are measured at the same spacial point; in the proper frame of test mass $a$ this point is located at $x^{\prime}=0$ where rate of the built-in-test-mass- $a$ clock coincides exactly with the rate of the laboratory clock which ticks identically everywhere. Therefore, both the clocks measure equal time intervals.

\section{FORWARD-TRIP POSITION METER}

Let us now analyze the operation of the forward trip position meter illustrated in Fig. 2. The system under consideration consists of only two test masses: laser (test mass $a$ ) and detector (test mass $b$ ) separated by a distance $L$ in the state of rest. Both the test masses have the built-in clocks, which initially (at the state of rest) are assumed to be perfectly synchronized. Laser emits the light wave and detector measures the phase shift with respect to its clock. Such a position meter (with slight modifications) might be a constituent part of Mach-Zehnder interferometer. Similarly to the previous section we will perform the analysis in both the laboratory and detector frames and compare the results.

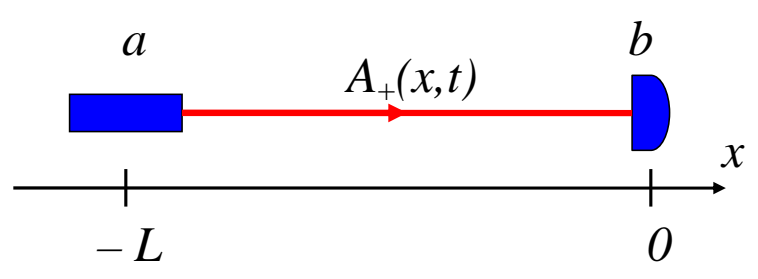

FIG. 2: A forward-trip position meter. Initially at the state of rest the built-in clocks in both the laser (test mass $a$ ) and detector (test mass $b$ ) are assumed to be perfectly synchronized. Detector measures the phase shift of light wave emitted by laser. In the state of rest the distance between the test masses equals to $L$.

\section{A. Analysis in the inertial frame}

Let the coordinates of the test masses be $x_{a}(t)=$ $-L+\xi_{a}(t)$ and $x_{b}(t)=0+\xi_{b}(t)$. Similarly to the previous section we write the wave emitted by laser in the following form (see Appendix $B$ for the account of optical noise):

$$
A_{+}(x, t)=A_{+0} \exp \left\{-i \omega_{0}\left[t-\frac{x-x_{a}(t-(x+L) / c)}{c}\right]\right\} .
$$

Detection of the wave and measurement of its phase 
takes place at $x=x_{b}(t)=\xi_{b}(t)$ :

$A_{+}\left(x_{b}(t), t\right)=A_{+0} \exp \left\{-i \omega_{0}\left[t-\frac{x_{b}(t)-x_{a}(t-\tau)}{c}\right]\right\}$.

Obviously, total variation of the wave equals to

$$
\delta a(t)=A_{+0} e^{i \omega_{0} \tau} i k_{0}\left[\xi_{b}(t)-\xi_{a}(t-\tau)\right] .
$$

The phase shift due to the test masses motion is

$$
\delta \Psi(t)=k_{0}\left[\xi_{b}(t)-\xi_{a}(t-\tau)\right] .
$$

Let the system move as the entire body, $\xi_{a}(t)=\xi_{b}(t)$. It is interesting that the first non-vanishing term in the expansion of $\delta \Psi(t)$ into series of $\tau$ is $k_{0} \dot{\xi}_{b}(t) \tau$, i.e. is proportional to the instantaneous velocity of the body. This does not mean, however, that one is able to measure the latter, contradicting the relativity principle. This result simply tells us that the phase shift (16) is unmeasurable by detector. In order to make it measurable we should perform a coordinate transformation that brings us from the laboratory frame to the frame of detector - the inverse transformation of Eqs. (11). However, from the logical point of view it is more convenient to perform the analysis completely in the frame of detector.

\section{B. Analysis in the non-inertial frame}

In the reference frame of detector (test mass $b)$ coordinates of the test masses are $x_{a}^{\prime}(t)=-L+\delta x_{a}^{\prime}(t)$ and $x_{b}^{\prime}(t)=0$.

From the viewpoint of detector laser emits electromagnetic wave described by the following vector-potential:

$$
\begin{aligned}
& A_{+}^{\prime}\left(x^{\prime}, t^{\prime}\right) \\
& =A_{+0}\left[1+w_{+}^{\prime}\left(x^{\prime}, t^{\prime}\right)-w_{+}^{\prime}\left(-L, t^{\prime}-\left(x^{\prime}+L\right) / c\right)\right] \\
& \quad \times \exp \left\{-i \omega_{0}\left[t^{\prime}-\frac{x^{\prime}-x_{a}^{\prime}\left(t^{\prime}-\left(x^{\prime}+L\right) / c\right)}{c}\right]\right\} .
\end{aligned}
$$

This corresponds to the boundary condition which states that at the point of laser location $A_{+}\left(x_{a}^{\prime}(t), t^{\prime}\right)=$ $A_{+0} e^{-i \omega_{0} t^{\prime}}$. In other words, in the immediate vicinity of the laser light wave acquires neither localized phase shift due to laser motion nor the distributed phase shift due to the acceleration of the observer.

At the detector location:

$$
\begin{aligned}
A_{+}\left(x_{b}^{\prime}(t), t\right)= & A_{+0}\left[1+w_{+}^{\prime}(0, t)-w_{+}^{\prime}(-L, t-\tau)\right] \\
& \times \exp \left\{-i \omega_{0}\left[t^{\prime}-\frac{x_{b}^{\prime}(t)-x_{a}^{\prime}(t-\tau)}{c}\right]\right\} .
\end{aligned}
$$

Since $x_{b}^{\prime}(t)=0$ and $w_{+}^{\prime}(0, t)=0$, total variation of the optical field equals to:

$$
\delta a^{\prime}(t)=-A_{+0} e^{i \omega_{0} \tau}\left[i k_{0} \delta x_{a}^{\prime}(t-\tau)+w_{+}^{\prime}(-L, t-\tau)\right] .
$$

The phase shift we are interested in equals to:

$$
\begin{aligned}
& i \delta \Psi^{\prime}(t)=-i k_{0} \delta x_{a}^{\prime}(t-\tau)-w_{+}^{\prime}(-L, t-\tau) \\
& =-i k_{0}\left[\delta x_{a}^{\prime}(t-\tau)+\dot{\xi}_{b}(t-\tau) \tau+\xi_{b}(t-\tau)-\xi_{b}(t)\right] .
\end{aligned}
$$

If $\delta x_{a}^{\prime}(t)=0$ we obtain: $\delta \Psi^{\prime}(t) \approx k_{0} \ddot{\xi}_{b}(t) \tau^{2} / 2$. This result can be qualitatively explained in the following way. If $\ddot{\xi}_{b}(t)>0$ then the photon moves against the direction of effective gravitational field with acceleration of the free fall $g_{\text {eff }}=-\ddot{\xi}_{b}(t)<0$. Thus, photon velocity is effectively reduced and the optical length increases from $L$ to $L+\left|\ddot{\xi}_{b}(t) \tau^{2} / 2\right|$. If $\ddot{\xi}_{b}(t)<0$ than the photon velocity is increased and optical length is reduced to $L-\left|\ddot{\xi}_{b}(t) \tau^{2} / 2\right|$.

Substituting into the obtained phase shift solution (6) for $\delta x_{a}^{\prime}(t)=\xi_{a}(t)-\xi_{b}(t)$, we obtain:

$$
\delta \Psi^{\prime}(t)=k_{0}\left[\xi_{b}(t)-\xi_{a}(t-\tau)-\dot{\xi}_{b}(t-\tau) \tau\right] .
$$

Comparing this result with formula (16) we conclude that the last term in square brackets, $-\dot{\xi}_{b}(t-\tau) \tau$, recovers an agreement with the relativity principle. This term describes the difference in the proper rate of clock in laser at $x=-L$ and detector at $x=0$. From the viewpoint of laboratory observer both clocks tick identically.

\section{Physical reason of discrepancy between the reference frames}

It is useful to consider the physical reason underlying the discrepancy of results obtained in the laboratory frame and the proper frame of detector.

Consideration in the laboratory frame implies that all the clocks built in the test masses are synchronized with the laboratory clock. In turn, this requires the knowledge of the velocities of the test masses with respect to the laboratory frame. Evidently, an observer, staying in rest in the laboratory, is able to measure the velocities of the test masses with respect to the latter. In fact, one may straightforwardly derive from special relativity that phase shift (16) is the one measured by the laboratory observer who, therefore, is able to measure the common speed of the test masses.

On the other hand, there is no any "external observer" in a system of $N$ test masses. In such a system test masses are able to synchronize their clocks only with respect to each other. Evidently, the absence of knowledge of the test masses velocities with respect to the laboratory will result in the accuracies of the order of $(v / c) L$ in clock synchronization. The latter will inevitably enter the phase shift as $k_{0}(v / c) L$ which is exactly the term missing in (16) and present in (17). Therefore, we conclude that the discrepancy between two reference frames lies in different procedures of clocks synchronization.

From these reasonings it is also clear why calculations of the round-trip scheme in both the frames produce identical results. 


\section{SPACE-TIME OF AN ACCELERATED OBSERVER WITH ACCOUNT FOR GRAVITATIONAL WAVES}

The performed analysis can be straightforwardly generalized to take into account the action of the GWs on position meters. According to Refs. [11, 13] inertial and gravitational effects do not couple in the first order. Therefore, one may "linearly combine" results of this paper with results of Ref. 12 to calculate the response of position meter to GWs in the proper reference frame of detector. For instance, consider space-time metric

$$
\begin{aligned}
d s^{2}= & -\left(c d t^{\prime}\right)^{2}\left[1+\frac{2}{c^{2}} a_{x}\left(t^{\prime}\right) x^{\prime}\right]+d x^{\prime 2}+d y^{\prime 2}+d z^{\prime 2} \\
& +\frac{1}{2} \frac{x^{\prime 2}-y^{\prime 2}}{c^{2}} \ddot{h}\left(t^{\prime}-z^{\prime} / c\right)\left(c d t^{\prime}-d z^{\prime}\right)^{2}
\end{aligned}
$$

corresponding to the observer moving with non-geodesic acceleration $a_{x}\left(t^{\prime}\right)$ along the $x$-axis in the field of weak plane gravitational wave $h\left(t^{\prime}-z^{\prime} / c\right)$ propagating along the $z$-axis normal to the $x y$-plane. In this section we will call the proper reference frame of an observer the local Lorentz (LL) gauge, since at $x^{\prime}=y^{\prime}=0$ metric is locally flat. Transverse-traceless (TT) gauge corresponds then to the laboratory frame (see below).

If the coordinate of the test mass relative to an observer equals to $X_{0}$ on average (in the state of rest) then the former moves according to the motion law

$$
\delta x^{\prime}(t)=\frac{1}{2} X_{0} h(t)+\xi(t)-\xi_{\mathrm{ref}}(t)
$$

where $\ddot{\xi}_{\text {ref }}(t)=a_{x}(t)$. Remind, that the difference between laboratory time and observer time leads to the negligible 2nd order effects.

Vector-potential of the electromagnetic wave propagating in this space-time along the $x$-axis can be written in the following form:

$$
A_{ \pm}^{\prime}\left(x^{\prime}, t^{\prime}\right)=A_{ \pm 0}\left[1+g_{ \pm}^{\prime}\left(x^{\prime}, t^{\prime}\right)+w_{ \pm}^{\prime}\left(x^{\prime}, t^{\prime}\right)\right] e^{-i\left(\omega_{0} t^{\prime} \mp k_{0} x^{\prime}\right)}
$$

where

$$
\begin{aligned}
& g_{ \pm}^{\prime}\left(x^{\prime}, t^{\prime}\right) \approx g_{ \pm}^{\prime}(x, t) \\
& =i k_{0}\left[\frac{1}{4} x \dot{h}(t) \frac{x}{c} \mp \frac{1}{2} x h(t)+\frac{c}{2} \int_{t \mp x / c}^{t} h\left(t_{1}\right) d t_{1}\right] \\
& w_{ \pm}^{\prime}\left(x^{\prime}, t^{\prime}\right) \approx w_{ \pm}^{\prime}(x, t) \\
& =i k_{0}\left[-\dot{\xi}_{\text {ref }}(t) \frac{x}{c} \pm \xi_{\text {ref }}(t) \mp \xi_{\text {ref }}(t \mp x / c)\right] .
\end{aligned}
$$

If one considers, for instance, the response of a roundtrip position meter to GW and fluctuative motions of the test masses using the method developed in Sec. IV] the obtained phase shift will be:

$\delta \Psi_{\text {r.t. }}^{\mathrm{TT}}(t)=\delta \Psi_{\text {r.t. }}^{\mathrm{LL}}(t)$

$$
=k_{0}\left[2 \xi_{b}(t-\tau)-\xi_{a}(t)-\xi_{a}(t-2 \tau)\right]+\frac{\omega_{0}}{2} \int_{t-2 \tau}^{t} h\left(t_{1}\right) d t_{1} .
$$

Note that this result can be derived in both the TT and LL gauges. For small enough $\tau(\Omega \tau \ll 1$ in spectral domain) $\delta \Psi_{\text {r.t. }} \approx 2 k_{0}\left(L h / 2+\xi_{b}-\xi_{a}\right)$. This is the common result for the LIGO-type GW detectors, where $\xi_{a, b}(t)$ are the fluctuative displacements of the test masses that mimic the GW signal $h(t)$.

For a forward-trip coordinate meter corresponding phase shift, calculated in the LL gauge, will be:

$$
\begin{aligned}
\delta \Psi_{\mathrm{f.t.}}^{\prime \mathrm{LL}}(t)= & k_{0}\left[\xi_{b}(t)-\xi_{a}(t-\tau)-\dot{\xi}_{b}(t-\tau) \tau\right] \\
& -\frac{1}{4} k_{0} L \dot{h}(t-\tau) \tau+\frac{\omega_{0}}{2} \int_{t-\tau}^{t} h\left(t_{1}\right) d t_{1} .
\end{aligned}
$$

Expanding into series of $\tau$ we obtain $\delta \Psi_{\text {f.t. }} \approx k_{0}(L h / 2+$ $\left.\xi_{b}-\xi_{a}\right)$ which is exactly the half of the round-trip phase.

Note that the phase shift (18) differs from the one that could be obtained in the TT gauge:

$$
\delta \Psi_{\text {f.t. }}^{\mathrm{TT}}(t)=k_{0}\left[\xi_{b}(t)-\xi_{a}(t-\tau)\right]+\frac{\omega_{0}}{2} \int_{t-\tau}^{t} h\left(t_{1}\right) d t_{1} .
$$

Comparing this phase shift with the phase shift (16) we may conclude that the TT gauge in GW physics plays the similar role to the laboratory frame in the globally flat space-time. For instance, since $g_{00}(x, t) \equiv-1$ in the TT gauge [2, 3, 4], clock tick identically everywhere. GW manifests itself as the effective time-dependent optical refraction index; test masses stay in rest in this gauge. The proper reference frame of detector corresponds then to the LL gauge, where rate of the clock coincides with the TT-clock only at the coordinate origin, and GW manifests itself as the tidal force-field acting on the test masses; electromagnetic wave is affected only slightly. Therefore, (in full similarity with Newtonian physics) it is natural that the results obtained in different gauges do not coincide in accordance with different procedures of clocks synchronization associated with them as described in the previous Section.

It will be also interesting to examine how additional term, $-k_{0} L \dot{h}(t-\tau) \tau / 4$, in Eq. (18) influences the responses of Mach-Zehnder or LISA-type 14] interferometers. This problem requires additional detailed analysis and we do not consider it in this paper.

The related problem is the transformation of results between different proper reference frames when analyzing an array of emitters and receivers. In general this should be performed by the coordinate transformation from one proper frame to another. However, such a transformation results in much more cumbersome calculations than performing the analysis for another proper frame from the beginning. 


\section{CONCLUSION}

In this paper we developed a method of analyzing the operation of the optical position meters in the reference frames of their detectors, which are non-inertial in general. First we studied the motion of the test masses and propagation of electromagnetic waves in the space-time of accelerated observer. Then we considered the operation of the round-trip position meter and found that the phase shift of light wave calculated in the laboratory (globally inertial) frame equals to the one calculated in the proper (non-inertial) reference frame of detector. This coincidence owes to the particular geometry of the round-trip scheme: phases of both the emitted and detected light waves are measured at the same spacial point by one clock. However, for the forward-trip position meter situation is completely different: the rate of laser and detector clocks, separated by a large distance, differ greatly. This results in different phase shifts calculated in the laboratory frame and the frame of detector. Namely, the former one contradicts the relativity principle and thus is unmeasurable.

We also discussed the generalization of the developed method to take into account the action of GWs. We demonstrated that the responses of the round-trip position meter, calculated in the TT and LL gauges coincide, while the ones of the forward-trip position meter differ, in full similarity with the laboratory and detector frames. The performed analysis could be useful in consideration of the various types of displacement-noise-free GW detectors, where fluctuative motion of detector may play the crucial role.

\section{Acknowledgments}

The author would like to thank S.P. Vyatchanin, F.Ya. Khalili and K. Somiya for valuable critical remarks on the paper.

This work was supported by LIGO team from Caltech and in part by NSF and Caltech grant PHY-0353775 and by Grant of President of Russian Federation NS5178.2006.2.

\section{APPENDIX A: SOLUTION OF THE WAVE EQUATION}

In this Appendix we solve the 1st order wave equation (9). For briefness we omit all the primes here:

$$
\frac{1}{c^{2}} \frac{\partial^{2} A_{ \pm}^{(1)}}{\partial t^{2}}-\frac{\partial^{2} A_{ \pm}^{(1)}}{\partial x^{2}}=\frac{a x}{c^{2}}\left(\frac{1}{c^{2}} \frac{\partial^{2} A_{ \pm}^{(0)}}{\partial t^{2}}+\frac{\partial^{2} A_{ \pm}^{(0)}}{\partial x^{2}}\right)
$$

$$
-\frac{\dot{a} x}{c^{3}} \frac{1}{c} \frac{\partial A_{ \pm}^{(0)}}{\partial t}+\frac{a}{c^{2}} \frac{\partial A_{ \pm}^{(0)}}{\partial x}
$$

Remind, that the 0th order solution is given by formula (8) and we omit the "c.c." terms.

It is convenient to solve this equation in spectral domain. Applying the theorem of convolution to the right side of the equation we obtain the 1st order equation in spectral domain:

$$
\begin{aligned}
& -\frac{\Omega^{2}}{c^{2}} A_{ \pm}^{(1)}(x, \Omega)-\frac{\partial^{2} A_{ \pm}^{(1)}(x, \Omega)}{\partial x^{2}}= \\
& =-\frac{k_{0} A_{ \pm 0} e^{ \pm i k_{0} x}}{c^{2}} a\left(\Omega-\omega_{0}\right)\left[2 k_{0} x-\frac{\Omega-\omega_{0}}{c} x \mp i\right]
\end{aligned}
$$

Let us introduce the following notations:

$$
A_{ \pm}^{(1)}(x, \Omega)=A_{ \pm}(x), \quad \frac{\Omega}{c}=k, \quad \frac{A_{ \pm 0} a\left(\Omega-\omega_{0}\right)}{c^{2}}=B .
$$

In this notation equation takes the following form:

$$
\frac{d^{2} A_{ \pm}}{d x^{2}}+k^{2} A_{ \pm}=B e^{ \pm i k_{0} x}\left(3 k_{0}^{2} x-k_{0} k x \mp i k_{0}\right)
$$

We solve this equation with the method of variation of constants (see any ODE handbook): $A_{ \pm}(x)=$ $C_{ \pm 1}(x) e^{i k_{0} x}+C_{ \pm 2}(x) e^{-i k_{0} x}$. The set of equations for $C_{ \pm 1,2}$ is:

$$
\begin{aligned}
& \frac{d C_{ \pm 1}}{d x} e^{i k x}+\frac{d C_{ \pm 2}}{d x} e^{-i k x}=0 \\
& i k \frac{d C_{ \pm 1}}{d x} e^{i k x}-i k \frac{d C_{ \pm 2}}{d x} e^{-i k x}=B e^{ \pm i k_{0} x}\left(3 k_{0}^{2} x-k_{0} k x \mp i k_{0}\right) .
\end{aligned}
$$

Straightforward integration leads to:

$$
C_{+1}(x)=C_{+10}+\frac{B}{2 k\left(k_{0}-k\right)^{2}} e^{i\left(k_{0}-k\right) x}\left[x\left(-3 k_{0}^{2}+4 k_{0}^{2} k-k_{0} k^{2}\right)-2 i k_{0}^{2}\right],
$$




$$
\begin{aligned}
& C_{+2}(x)=C_{+20}+\frac{B}{2 k\left(k_{0}+k\right)^{2}} e^{i\left(k_{0}+k\right) x}\left[x\left(3 k_{0}^{2}+2 k_{0}^{2} k-k_{0} k^{2}\right)+\left(2 i k_{0}^{2}-2 i k_{0} k\right)\right], \\
& C_{-1}(x)=C_{-10}+\frac{B}{2 k\left(k_{0}+k\right)^{2}} e^{-i\left(k_{0}+k\right) x}\left[x\left(3 k_{0}^{2}+2 k_{0}^{2} k-k_{0} k^{2}\right)+\left(-2 i k_{0}^{2}+2 i k_{0} k\right)\right], \\
& C_{-2}(x)=C_{-20}+\frac{B}{2 k\left(k_{0}-k\right)^{2}} e^{-i\left(k_{0}-k\right) x}\left[x\left(-3 k_{0}^{2}+4 k_{0}^{2} k-k_{0} k^{2}\right)+2 i k_{0}^{2}\right] .
\end{aligned}
$$

Constants of integration are derived from the boundary condition (10, 11):

$$
\begin{aligned}
& A^{(1)}(x, \Omega)=A_{+}^{(1)}(x, \Omega)+A_{-}^{(1)}(x, \Omega), \\
& A_{+}^{(1)}(0, \Omega)=A_{-}^{(1)}(0, \Omega)=0 .
\end{aligned}
$$

Therefore, we obtain the following solution of the 1st order in spectral domain:

$$
\begin{aligned}
A_{ \pm}^{(1)}(x, \Omega)= & \frac{1}{c^{2}} A_{ \pm 0} e^{ \pm i k_{0} x} a\left(\Omega-\omega_{0}\right) \\
& \times\left\{\eta(\Omega) x \pm i c \zeta(\Omega)\left[1-e^{\left(\Omega / c-k_{0}\right) x}\right]\right\}
\end{aligned}
$$

where

$$
\begin{aligned}
& \eta(\Omega)=\frac{-3 \omega_{0}^{4}+\omega_{0}^{3} \Omega+3 \omega_{0}^{2} \Omega^{2}-\omega_{0} \Omega^{3}}{\left(\omega_{0}-\Omega\right)^{2}\left(\omega_{0}+\Omega\right)^{2}}, \\
& \zeta(\Omega)=\frac{-5 \omega_{0}^{3}+2 \omega_{0}^{2} \Omega-\omega_{0} \Omega}{\left(\omega_{0}-\Omega\right)^{2}\left(\omega_{0}+\Omega\right)^{2}} .
\end{aligned}
$$

Let us introduce the notation:

$$
\begin{aligned}
w_{ \pm}(x, \Omega)= & \frac{1}{c^{2}} a\left(\Omega-\omega_{0}\right) \\
& \times\left\{\eta(\Omega) x \pm i c \zeta(\Omega)\left[1-e^{ \pm\left(\Omega / c-k_{0}\right) x}\right]\right\} .
\end{aligned}
$$

Then

$$
\begin{aligned}
A_{ \pm}^{(1)} & (x, t)=\int_{-\infty}^{+\infty} A_{ \pm 0} w_{ \pm}(x, \Omega) e^{ \pm i k_{0} x} e^{-i \Omega t} \frac{d \Omega}{2 \pi} \\
& =A_{ \pm 0} e^{-i\left(\omega_{0} t \mp k_{0} x\right)} \int_{-\infty}^{+\infty} w_{ \pm}\left(x, \Omega+\omega_{0}\right) e^{-i \Omega t} \frac{d \Omega}{2 \pi} .
\end{aligned}
$$

It is straightforward to verify that in the $\Omega \ll \omega_{0}$ limit

$$
\eta\left(\Omega+\omega_{0}\right) \approx \frac{\omega_{0}}{\Omega}, \quad \zeta\left(\Omega+\omega_{0}\right) \approx-\frac{\omega_{0}}{\Omega^{2}},
$$

and

$$
w_{ \pm}\left(x, \Omega+\omega_{0}\right)=\frac{a(\Omega)}{\Omega^{2}}\left[\frac{\Omega}{c} k_{0} x \mp i k_{0}\left(1-e^{ \pm i \Omega x / c}\right)\right] .
$$

Remind now, that $a(\Omega) / \Omega^{2}=-\xi_{\text {ref }}(\Omega)$. Ultimately, in time domain we obtain the following solution:

$$
A_{ \pm}^{(1)}(x, t)=A_{ \pm 0} w_{ \pm}(x, t) e^{-i\left(\omega_{0} t \mp k_{0} x\right)},
$$

$$
\begin{aligned}
w_{ \pm}(x, t) & =\int_{-\infty}^{+\infty} w_{ \pm}\left(x, \Omega+\omega_{0}\right) e^{-i \Omega t} \frac{d \Omega}{2 \pi} \\
& =-i k_{0} \dot{\xi}_{\text {ref }}(t) \frac{x}{c} \pm i k_{0}\left[\xi_{\text {ref }}(t)-\xi_{\text {ref }}(t \mp x / c)\right] .
\end{aligned}
$$

Remind, that the prime should be inserted everywhere, since we work in the non-inertial frame.

APPENDIX B: INFLUENCE OF THE OPTICAL NOISE

In this Appendix we briefly consider the influence of laser optical noise on the responses of round- and forward-trip position meters.

In general optical noise can be taken into account by adding the following term to initial vector-potentials [12]:

$$
a_{+}(x, t)=\int_{-\infty}^{+\infty} a_{+}\left(\omega_{0}+\Omega\right) e^{-i \Omega\left(t-\frac{x-x_{0}}{c}\right)},
$$

where $x_{0}$ is the reference point for specific problem. For instance, for the round-trip meter $x_{0}=0$ and for the forward-trip meter $x_{0}=-L$. Since optical noise, in practice, is comparable to other noises in their magnitude, one can neglect the interaction between GW and acceleration fields with it.

Now substituting optical noise into the corresponding boundary problems we obtain in spectral domain:

$$
\begin{aligned}
\delta a\left(\omega_{0}+\Omega\right)= & -a_{+}\left(\omega_{0}+\Omega\right) e^{2 i\left(\omega_{0}+\Omega\right) \tau} \\
& +A_{+0} e^{2 i \omega_{0} \tau} i k_{0}\left[\xi_{a}-2 \xi_{b} e^{i \Omega \tau}+\xi_{a} e^{2 i \Omega \tau}\right],
\end{aligned}
$$

for the round-trip meter in both the laboratory and proper frame of detector;

$$
\begin{aligned}
\delta a\left(\omega_{0}+\Omega\right)= & a_{+}\left(\omega_{0}+\Omega\right) e^{i\left(\omega_{0}+\Omega\right) \tau} \\
& +A_{+0} e^{i \omega_{0} \tau} i k_{0}\left[\xi_{b}-\xi_{a} e^{i \Omega \tau}\right],
\end{aligned}
$$

for the forward-trip meter in the laboratory frame and

$$
\begin{aligned}
\delta a\left(\omega_{0}+\Omega\right)= & a_{+}\left(\omega_{0}+\Omega\right) e^{i\left(\omega_{0}+\Omega\right) \tau} \\
& +A_{+0} e^{i \omega_{0} \tau} i k_{0}\left[\xi_{b}-\xi_{a} e^{i \Omega \tau}+i \Omega \tau \xi_{b} e^{i \Omega \tau}\right],
\end{aligned}
$$

for the forward-trip meter in the proper frame of detector. Here $\xi_{a, b}=\xi_{a, b}(\Omega)$. GW can be taken into account straightforwardly. 
[1] LIGO website, URL http://www.ligo.caltech.edu.

[2] C. Misner, K. Thorne and J. Wheeler, Gravitation, vol. 3 (San Francisco, W.H. Freeman and Company, 1973).

[3] R. Blandford and K.S. Thorne, $P h$ 136: Applications of Classical Physics (California Institute of Technology, Pasadena, 2003), chap. 26, URL http://www.pma.caltech.edu/Courses/ph136/yr2002/chap2

[4] E.E. Flanagan and S.A. Hughes, New J. Phys. 7, 204 (2005), arXiv:gr-qc/0501041v3.

[5] S. Kawamura and Y. Chen, Phys. Rev. Lett. 93, 211103 (2004), arXiv:gr-qc/0405093v2.

[6] Y. Chen and S. Kawamura, Phys. Rev. Lett. 96, 231102 (2006), arXiv:gr-qc/0504108v3.

[7] Y. Chen et al., Phys. Rev. Lett 97, 151103 (2006), arXiv:gr-qc/0603054v2.
[8] K. Somiya et al., Phys. Rev. D 76, 022002 (2007).

[9] S.P. Tarabrin and S.P. Vyatchanin, submitted to Phys. Rev. D, arXiv:gr-qc/0804.3955.

[10] R. Blandford and K.S. Thorne, $P h$ 136: Applications of Classical Physics (California Institute of Technology, Pasadena, 2003), chap. 23, URL

26/CBe6p1/.pd佇.pma.caltech.edu/Courses/ph136/yr2002/chap23/0223

[11] W.-T. Ni and M. Zimmermann, Phys. Rev. D 17, 1473 (1978).

[12] S.P. Tarabrin, Phys. Rev. D 75, 102002 (2007), arXiv:grqc/0701156v2.

[13] K.-P. Marzlin, Phys. Rev. D 50, 888 (1994).

[14] C. Cutler, Phys. Rev. D 57, 7089 (1998), arXiv:grqc/9703068. 